\title{
e-Interview
}

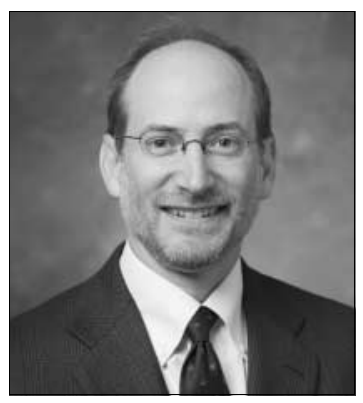

\section{John H. Krystal}

John H. Krystal is Robert L. McNeil Jr. Professor of Translational Research and Chair of the Department of Psychiatry of the Yale University School of Medicine. He trained at Yale Medical School and the Yale Psychiatry Residency Program. His special interest is translational neuroscience.

If you were not a psychiatrist, what would you do?

I have been a psychiatrist for over 20 years and being a psychiatrist is an integral part of my identity. If I pursued other endeavors at this point, I would do so as a psychiatrist.

What has been the greatest impact of your profession on you personally? Overall, the work has been fun, satisfying, challenging and stimulating. I am extremely grateful to have stumbled upon such a rewarding career.

\section{Do you feel stigmatised by your} profession?

I do not feel stigmatised, but I find that I am frequently in settings where people need to be reminded about the magnitude of the impact of psychiatric disorders and the contributions of psychiatry and psychiatric research.

What are your interests outside of work? Outside of work, my family is my top priority. I also enjoy music and sports (tennis, skiing, hiking), particularly opportunities to enjoy these activities with friends.

\section{What job gave you the most useful} training experience?

Perhaps my initial job, creating and then leading a schizophrenia research unit and neurobiological studies unit at the West Haven VA Medical Center.
How has the political environment influenced your work?

I think that economic rather than political factors have profoundly affected my work. Tight resources have limited the type of work that I do to highly specialised research settings.

\section{What part of your work gives you the} most satisfaction?

As an investigator, I enjoy conducting studies that have the possibility of definitively answering questions, particularly testing favourite hypotheses. As an administrator, I love helping to create opportunities for young faculty to realise their potential. As a clinician, watching a patient improve is enormously satisfying.

\section{What do you least enjoy?}

Administrative paperwork.

\section{What is the most promising opportunity} facing the profession?

New insights into brain function, the genome/epigenome, and the function of neural systems may provide, for the first time, a rational basis for the development of new treatments for psychiatric and addictive disorders.

\section{What is the greatest threat?}

There are serious threats to the viability of the academic research enterprise related to the convergence of reduced investment in the psychiatric drug development by the pharmaceutical industry, limited growth in the societal (state, federal, research foundations) investment in psychiatry research, limited contexts for effective translational research within academia, and the significant increase in the expense of conducting research.

What single change would substantially improve quality of care?

Increasing the investment in psychiatry research.

What is the role of the psychiatrist in countries emerging from conflict? To provide care for patients and to act as advocates for people who's lives are affected by stress-related psychiatric disorders.

What is the most important advice you could offer to a new trainee?

Trainees should view their residency as a tool that they can use to learn what they need to learn rather than as an indoctrination process. Trainees with passionate interests should pursue these interests, to the extent possible, from the start of their residencies. For those without this sort of passion, I recommend trying things out that seem interesting as opposed to waiting for passions to emerge.

What is the role of the psychiatrist in rebuilding healthcare systems?

To design into the systems access to appropriate psychiatric assessment and preventive, therapeutic and rehabilitative treatments.

What single area of psychiatric practice is most in need of development?

Our field has come a long way in refining our diagnostic system and therapeutic approaches. Yet, nearly all aspects of our profession need fundamental evolution. We are an old field but a young science. Our diagnostic system is symptom based and disconnected from its genetic and neural substrates. Our pharmacotherapies are essentially palliative, ineffective for many patients, and not based on an understanding of the causes of the illnesses that we treat. We are just now, for the first time, combining some medications and psychotherapies on the basis of an understanding of specific neural mechanisms as opposed to because they both 'help'. Beyond symptoms, we have no biomarkers to aid the individual clinician in making diagnoses, choosing a medication, or to monitor treatment efficacy. In many areas, though, psychiatry is making significant progress; making psychiatry one of the most exciting areas within medicine.

What single area of psychiatric research should be given priority?

Translational genetics that has the potential of identifying relatively rare gene variations or epigenetic modifications that have large effects on the development of phenotypes related to psychiatry.

How would you like to be remembered? With regard to my work, I am too absorbed by what I hope to do rather than by what I have done to give this question much thought.

Dominic Fannon

doi: 10.1192/pb.bp.110.032805 